\title{
PROPYLTHIOURACIL-INDUCED ANTINEUTROPHIL CYTOPLASMIC ANTIBODY-ASSOCIATED VASCULITIS
}

Marcela Miguens Castelar Pinheiro ${ }^{1, \star}$, Lucas Victória de Oliveira Martins ${ }^{1}$

1.Universidade Federal de São Paulo, São Paulo (SP), Brazil.

${ }^{\star}$ Corresponding author: marcelacastelar@yahoo.com.br

\section{BACKGROUND}

Propylthiouracil (PTU) is a common antithyroid drug, known to cause vasculitis associated with antineutrophil cytoplasmic antibodies (ANCA) (AAV) with multiple specificities of the ANCA antigen and a different course of the disease. In view of the high morbidity and mortality related to primary AAV, the identification of potentially reversible causes, such as medication, and prompt treatment is important.

\section{CASE REPORT}

A 62-year-old woman with a previous diagnosis of Graves' disease presented with weakness, unintentional weight loss (5 kg), fever, chills, night sweats and skin lesions. The symptoms started five months ago after switching methimazole for PTU. The medication switch was due to poor disease control. On examination, the patient had goiter, ocular proptosis and purpuric skin rash with necrotic center in the lower limbs (Fig. 1). Laboratory tests revealed pancytopenia (hemoglobin 6.7 g/dL; leukocytes 1,970/mm³; neutrophils $720 / \mathrm{mm}^{3}$; platelet count 35,000/mm3), elevated acute phase reactants (C-reactive protein $274 \mathrm{mg} / \mathrm{L}$; erythrocyte sedimentation rate $138 \mathrm{~mm} / \mathrm{h}$ ), normal renal function and urinalysis. The main hypothesis was adverse effect of PTU, due to pancytopenia (myelotoxic drug effect) and a possible systemic vasculitis (purpuric skin rash and constitutional symptoms). The diagnostic work-up included negative serum tests and culture for infectious diseases, no signs of upper or lower airway involvement on computed tomography scan and negative laboratory tests (antinuclear antibody, anti-dsDNA, anti-ENA and cryoglobulins). An ANCA test by indirect immunofluorescence was positive with cytoplasmic ANCA (c-ANCA) pattern with high titers (1/160). Skin biopsy showed leukocytoclastic vasculitis of small blood vessels without immune-complex deposition. The diagnosis of PTU-induced AAV was confirmed and the treatment included cessation of PTU and a short course of prednisone with resolution of constitutional symptoms, skin rash and pancytopenia. The patient underwent a total thyroidectomy to treat Graves' disease. Follow-up after one year, the patient is well, with no signs of vasculitis, without corticosteroids or immunosuppressors and controlled Graves' disease.

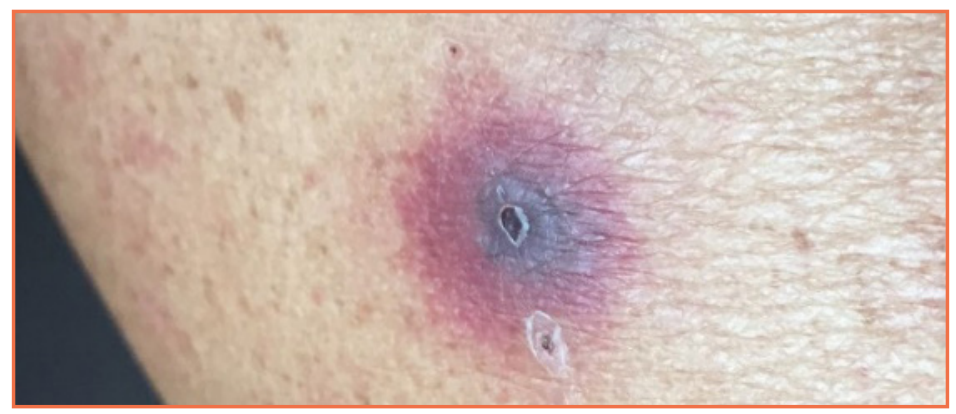

Figure 1. Purpuric skin rash with necrotic center.

\section{CONCLUSION}

Patients undergoing treatment with PTU should be monitored for signs of drug-induced AAV. Epidemiological studies show that $15-64 \%$ of patients receiving PTU develop ANCA positive and of ANCA positive patients $27.3 \%$ develops drug-induced AAV. Compared with primary AAV, PTU-induced AAV usually has a milder course with predominant constitutional symptoms and skin involvement and less visceral involvement. Early cessation of the disease-causing medication and appropriate immunosuppressive therapy is the standard treatment for drug-induced AAV, which is associated with a good prognosis. 